Global COE Hi-Stat Discussion Paper Series 186

$$
\begin{aligned}
& \text { Research Unit for Statistical } \\
& \text { and Empirical Analysis in Social Sciences (Hi-Stat) }
\end{aligned}
$$

\title{
Formation of Decentralized Manufacturer-Supplier Networked Market
}

Yasuhiro Shirata

April 2011

Hi-Stat 


\title{
Formation of Decentralized Manufacturer-Supplier Networked Market * Yasuhiro Shirata ${ }^{\dagger}$
}

April 12, 2011

\begin{abstract}
This paper studies trading in a two-sided market where firms strategically form a network. In a networked market, manufacturers and suppliers must be connected by links for trading. We show that if no contingent contract is available, then any pairwise Nash stable network is inefficient. Each supplier under-invests in links (a hold-up problem). If a contract contingent on direct links is available and link cost is low, then the under-investment problem solves. Furthermore, the complete network resulting in the Walrasian outcome is uniquely pairwise Nash stable. However, this outcome is also inefficient. A new hold-up problem, over-investment in links, arises.
\end{abstract}

\section{Introduction}

We often observe that firms build bilateral connections only with specific partners. In many industries, upstream firms and downstream firms invest in relation-specific assets such as the Internet, electric power lines, and pipelines to distribute their resources.

Such a bilateral relationship is called a link, and a pattern of links between upstream firms and downstream firms is called a network. Since each pair of firms must be linked for trading, architecture of networks will play a central role in the networked market. This paper examines whether decentralized trading in a manufacturer-supplier networked market with divisible goods achieves efficient and the competitive trading, comparing to the standard competitive market theory.

Recently, the bueyr-seller networked market has attracted much attention (Corominas-Bosch [4], Kranton and Minehart [9, 10]). For example, Kranton and Minehart [10] show that every efficient network is an equilibrium outcome. However, they studies centralized trading. It is assumed that an existence of centralized mechanisms; an auctioneer (Kranton and Minehart [10]) and a matchmaker (Corominas-Bosch [4]). These social planners can collect all messages and impose any trading using predetermined mechanisms. In a manufacturer-supplier networked market, however, firms

\footnotetext{
*I am very grateful to Professor Akira Okada for his guidance and encouragement. I also thank Kalyan Chatterjee, Daisuke Oyama, Fumitoshi Moriya, Nozomu Muto, Yasuhiro Arai, and participants in seminars at Hitotsubashi University, SWET 2008, 2010, and 14th DC Conference in Japan for helpful comments. This work is supported by Grant-in-Aid for Japan Society for the Promotion of Science (JSPS) Fellows.

${ }^{\dagger}$ Graduate School of Economics, Hitotsubashi University, 2-1 Naka, Kunitachi, Tokyo, 186-8601, Japan. E-mail: ed081004@g.hit-u.ac.jp
} 
are not anonymous, price-taking, and match-taking. Therefore, no such centralized mechanisms are available, and any trading is decentralized.

One of the seminal studies about the decentralized trading in a non-networked buyer-seller market is Rubinstein and Wolinsky [11]. They find that the Walrasian equilibrium trading between buyers and sellers is supported by a unique subgame perfect equilibrium if strategies for traders are restricted to stationary strategies. Gale and Sabourian [6] extend this result to a market with heterogeneous buyers and sellers. However, these studies cannot apply to the networked market since they assume that any buyer can be paired with any seller through the random matching.

Furthermore, the above studies of buyer-seller market assume unit demands and unit supplies. Each buyer demands one unit of (indivisible) goods and each seller owns one unit of goods. Thus, goods have to be traded through a one-to-one matching. Gale [5] points out that in the one-toone matching market, it is difficult to examine whether the results support the competitive market theory since the competitive outcome coincides with the monopolistic outcome.

Comparing to the buyers-seller market, it is natural that goods are traded through a many-tomany matching in a manufacturer-supplier networked market. Each supplier usually sell goods to multi manufacturers, and vice versa. Thus, we assume that divisible goods are traded by multi firms in a manufacturer-supplier networked market.

Therefore, we study decentralized trading in a manufacturer-supplier networked market with divisible goods. Our study focuses on two points to discover how network architecture affects on trading. The first is transaction cost. The competitive market theory assumes any trader can buy or sell goods to any other trader with no transaction cost. Then, the Walrasian equilibrium is always efficient. However, any firm must bear transaction costs to establish links in the networked manufacturer-supplier market. Then, the competitive market mechanism may implement an inefficient allocation (Coase [3], Williamson [13]). The second is to find a role of side-payment contracts for decentralized trading in the networked market. We compare a case where no side-payment contracts is available with a case where simple side-payment contracts contingent on links are available.

We first analyses a networked market consisting of two heterogeneous manufacturers, two heterogeneous suppliers, and one type of divisible goods. It is a minimal economy where any firm in each side is competitive. It is shown that in this small networked market, any stable outcome is inefficient, regardless of the presence of side-payment contracts. When all firms are symmetric, we further show that the result holds in a large networked market consisting of $n$ manufacturers and $n$ suppliers $(n \geq 3)$. The origin of the inefficiency is an under-investment problem if no sidepayment contract is available. In contrast, it is an over-investment problem if side-payment contracts are available.

The rest of the paper is organized as follows. Section 2 gives a formal definition of the networked market with divisible goods. Section 3 analyses the networked market by the competitive market theory as a benchmark. We introduce the notions of the competitive market theory into the 
networked market; the $g$-competitive equilibrium and the $g$-efficient allocation. We then show a version of the first fundamental theorem of welfare economics, i.e. any $g$-competitive equilibrium allocation is $g$-efficient.

Next, we construct a strategic trading game. The game has two stages, a network formation stage and a bargaining stage. Manufacturers and suppliers establish a network in the first stage and they trade on that network in the second stage. Section 4 constructs the bargaining game on a given network. We show that the unique linear subgame perfect equilibrium allocation is $g$-efficient for all $g$, but it is $g$-competitive if and only if the network is complete. If a supplier links with multi manufacturers then he obtain their surplus, otherwise he obtains no surplus.

Section 5 constructs a network formation game. All firms announce the set of partners with whom they want to link. Bilateral investments of a manufacturer and a supplier in relationshipspecific assets enable them to trade. Thus, any link in networks is established if and only if both a manufacturer and a supplier bilaterally agree to form it. We show that, in this link-announcement game, an empty and a supplier-monopolized network are pairwise Nash stable and any efficient network is not Nash stable.

Section 6 extends the link announcement game. Manufacturers and suppliers often contract with side-payments contingent on their links. Each firm can offer a contingent contract that (s)he pays transfer if a partner agrees with forming a link. When cost of forming a link is low, the complete networked market is pairwise Nash stable in this network formation game with direct transfer. In the complete networked market, however, the stable trading is inefficient since firms over-invest in links.

Section 7 analyses a large economy where there are $n$ symmetric manufacturers and $n$ symmetric suppliers. We show that any pairwise Nash stable outcome is inefficient in the large market. Section 8 concludes with several remarks.

\section{Networked Market}

This section introduces a two-sided networked market. There are $n$ manufacturers $I$ (indexed by $i=1,2, \ldots, n), n$ suppliers $J$ (indexed by $j=1,2, \ldots, n$ ), one type of resources (or intermediate goods) $x \in \mathbb{R}$, and money. We assume that their payoff functions are quasi-linear. Each supplier $j$ owns his endowment of resources $\omega_{j}>0$, and has no valuation over resources $x$.Each manufacturer $i$ has the following profit function $f^{i}: \mathbb{R} \rightarrow \mathbb{R}^{1}$

Assumption 1. For all $i \in I$, profit function $f^{i}$ is twice differentiable and

$1.1 f^{i}(0)=0$ and $\frac{d f^{i}}{d x^{i}}(0)=+\infty$,

$1.2 f^{i}$ is strictly increasing; $\frac{d f^{i}}{d x^{i}}>0$, and

\footnotetext{
${ }^{1}$ We can alternatively interpret each manufacturer $i$ and each supplier $j$ as a final consumer and a retailer, respectively. In this case, $f^{i}$ is $i^{\prime}$ s utility function.
} 


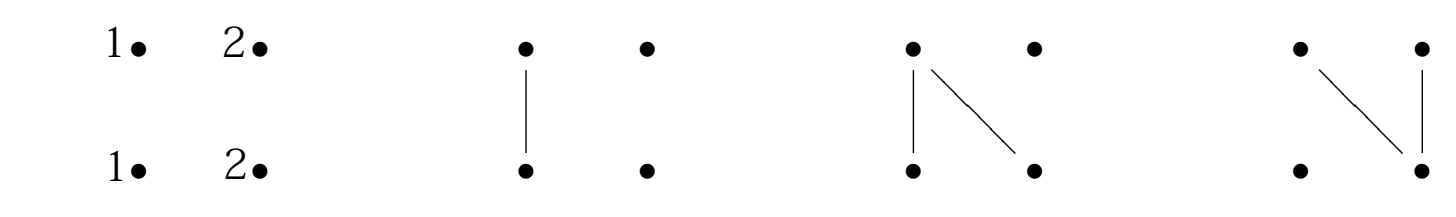
(a) network $g^{e}$
(b) network $g^{1}$
(c) network $g^{2}$
(d) network $g^{3}$

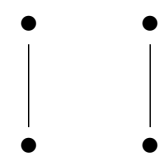

(e) network $g^{4}$

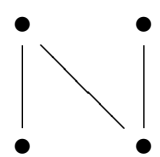

(f) network $g^{5}$

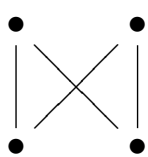

(g) network $g^{c}$

Figure 1: Feasible architecture of the $2 \times 2$ networked market. Manufacturers are in upper row and suppliers are in lower row.

$1.3 f^{i}$ is strictly concave; $\frac{d^{2} f^{i}}{d\left(x^{i}\right)^{2}}<0$.

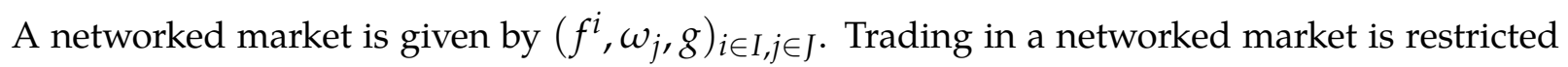
by network $g \subset\{i j\}_{i \in I, j \in J}$ which is a non-directed bipartite graph, where $i j$ is a non-directed link between manufacturer $i$ and supplier $j$. Figure 1 illustrates the set of all feasible types of networks $G$ when $n=2$. Let $\eta^{i}(g), \eta_{j}(g)$ be the number of links of manufacturer $i$, supplier $j$, and $L^{i}(g), L_{j}(g)$ be the set of agents linked with manufacturer $i$, supplier $j$, respectively. We denote the empty network $\left(\sum_{j=1}^{s} \eta_{j}\left(g^{e}\right)=0\right)$ by $g^{e}$, and the complete network $\left(\eta_{j}\left(g^{c}\right)=2\right.$ for all $\left.j\right)$ by $g^{c}$. Each manufacturer $i$ can buy resources from supplier $j$ if and only if they are linked $\left(j \in L^{i}(g)\right)$. In what follows, we abbreviate networked market $\left(f^{i}, \omega_{j}, g\right)_{i \in I, j \in J}$ as $g$ whenever no confusion arises.

\section{Benchmark: $g$-Competitive Equilibrium}

This section applies the competitive market mechanism to the networked market. We first define the competitive equilibrium in networked market $g$. Let $p_{j}^{i}(g)$ be a price when $i$ buys resource from $j$ in $g$, and $p(g)=\left(p_{1}^{1}(g), p_{2}^{1}(g), p_{1}^{2}(g), \ldots, p_{n}^{n}(g)\right)$.

Definition 1. An outcome $(\tilde{p}(g), \tilde{x}(g))$ is competitive relative to $g$ ( $g$-competitive) if

(i) for $i \in I,\left(\tilde{x}_{1}^{i}(g), \ldots, \tilde{x}_{n}^{i}(g)\right) \in \arg \max f^{i}\left(\sum_{j} x_{j}^{i}\right)-\sum_{j} \tilde{p}_{j}^{i}(g) x_{j}^{i}$ subject to $x_{j}^{i}=0$ for any ij $\notin g$, and

(ii) for $j \in J,\left(\tilde{x}_{j}^{1}(g), \ldots, \tilde{x}_{j}^{n}(g)\right) \in \arg \max \sum_{i} \tilde{p}_{j}^{i}(g) x_{j}^{i}$ subject to $\sum_{i} x_{j}^{i} \leq \omega_{j}$ and $x_{j}^{i}=0$ for any ij $\notin g$.

The $g^{\mathcal{C}}$-competitive equilibrium is the Walrasian equilibrium. 
We make two remarks. First, $\tilde{x}_{j}^{i}(g)$ can be negative. If $\tilde{x}_{j}^{i}(g)<0$, manufacturer $i$ sells resources to supplier $j$. However, $\sum_{j} \tilde{x}_{j}^{i}(g)>0$ for any $i$ with $\eta^{i}(g) \geq 1$ and any $g$ since any $i$ has zero endowments. Thus, any manufacturer $i$ can sell resources if $i$ has more than two links and buy resources from other suppliers.

Second, a non-uniform price vector conditional on links are allowed in $g$. However, any $g$ competitive equilibrium $(\tilde{p}(g), \tilde{x}(g))$ satisfies a uniform price among (directly or indirectly) connected agents. If it does not hold, then there is a firm who can arbitrage.

Let $\tilde{p}_{j}(g)$ be the competitive price for agents connected to supplier $j$. Thus, $\frac{d f^{i}}{d x_{j}^{i}}\left(\sum_{k} \tilde{x}_{k}^{i}(g)\right)=$ $\tilde{p}_{j}(g)$ for any $i \in L_{j}(g)$. We next show a version of the first fundamental theorem of welfare economics, i.e. any $g$-competitive equilibrium allocation is constrained efficient for all $g \in G$.

Definition 2. Let denote a (Pareto-)efficient allocation relative to $g$ ( $g$-efficient allocation) by $\hat{x}(g)=$ $\left(\hat{x}_{1}^{1}(g), \hat{x}_{2}^{1}(g), \hat{x}_{1}^{2}(g), \ldots, \hat{x}_{n}^{n}(g)\right)$. It is a solution of the following maximization problem:

$$
\begin{gathered}
\max _{x} \sum_{i \in I} f^{i}\left(\sum_{j} x_{j}^{i}\right) \\
\text { subject to } \sum_{i} x_{j}^{i} \leq \omega_{j} \text { for } j \in J, \\
x_{j}^{i}=0 \text { for all } i j \notin g .
\end{gathered}
$$

The solution $\hat{x}(g)$ is not unique generally. However, $\left(\sum_{j} \hat{x}_{j}^{1}(g), \ldots, \sum_{j} \hat{x}_{j}^{n}(g)\right)$ is unique by concavity of $f^{i}$. We denote the corresponding social welfare for $g$ by $\hat{\Pi}(g)=\sum_{i} f^{i}\left(\hat{x}^{i}(g)\right)$. For all $g, g^{\prime} \in G$, if $g \supset g^{\prime}$ then $\hat{\Pi}(g) \geq \hat{\Pi}\left(g^{\prime}\right)$.

Proposition 1. Any g-competitive equilibrium allocation is g-efficient.

Proof. For any $i j \notin g, x_{j}^{i}(g)=0$. Thus, if $L_{j}(g)=\varnothing$, then $\tilde{x}_{j}(g)=\hat{x}_{j}(g)=(0, \ldots, 0)$ for any $j \in J$.

Next, we consider the case where $L_{j}(g) \neq \varnothing$. Then, $\sum_{i} \tilde{x}_{j}^{i}(g)=\omega_{j}$ by condition (ii). Suppose that there is an allocation $y$ that (Pareto-)dominates $g$-competitive equilibrium allocation $\tilde{x}(g)$. Then, $y$ is infeasible since $\sum_{i} y_{j}^{i}>\sum_{i} \tilde{x}_{j}^{i}(g)=\omega_{j}$ for some $j$ by strict monotonicity of $f^{i}$. Therefore, any $g$-competitive equilibrium allocation is $g$-efficient.

Next, we define Consumer Surplus (CS) and Producer Surplus (PS) in the standard manner. For manufacturer $i \in I$, CS relative to $g$ for $i$ is given by

$$
C S^{i}(g)=f^{i}\left(\sum_{j} \hat{x}_{j}^{i}(g)\right)-\sum_{j} \tilde{p}_{j}(g) \hat{x}_{j}^{i}(g),
$$

and for supplier $j \in J$, PS relative to $g$ for $j$ is given by

$$
P S_{j}(g)=\tilde{p}_{j}(g) \sum_{i} \hat{x}_{j}^{i}(g)=\tilde{p}_{j}(g) \omega_{j}
$$


In the $g$-competitive equilibrium, each manufacturer $i$ obtains $C S^{i}(g)$ and each supplier $j$ obtains $P S_{j}(g)$. Note that $C S^{i}(g) \geq C S^{i}(g \backslash\{i j\})$ for any $g$ since $\sum_{l \in J} \hat{x}_{l}^{i}(g) \geq \sum_{l \in J \backslash\{j\}} \hat{x}_{l}^{i}(g \backslash\{i j\})$, and $P S_{j}(g) \geq P S_{j}(g \backslash\{i j\})$ for any $g$ since $\tilde{p}(g) \geq \tilde{p}(g \backslash\{i j\})$.

The game proceeds as follows: Manufacturers and suppliers form a trading network in the first stage (Network formation game). Then, they allocate resources and money given a network in the second stage (Bargaining game). We solve the game by backward induction.

\section{Bargaining Game in Networked Market}

We first analyses a small networked market with $n=2 .^{2}$ An economy is a bilateral duopoly, which is a minimal economy where any firm in each side is competitive. The set of feasible architecture of networks is given in Figure 1. For $g \in G \backslash\left\{g^{4}\right\}$, the $g$-competitive price is uniform and unique among all connected firms, given by $\tilde{p}(g)$. Thus, $\tilde{p}(g)=\frac{d f^{i}}{d x_{j}^{i}}\left(\sum_{k} \tilde{x}_{k}^{i}(g)\right)$ for $i, j=1,2$.

Protocol First, we formulate a bargaining protocol after network $g$ is formed. Given a network $g \in G$, each manufacturer $i$ proposes a payment schedule to supplier $j$ with $j \in L^{i}(g)$ contingent on an allotment from $j$ to $i, x_{j}^{i}$. A bargaining protocol is given by the following non-linear price competition:

Step 1: Every manufacturer $i$ bids payment schedule $t_{j}^{i}\left(x_{j}^{i}\right)$ to supplier $j$ for all $j \in L^{i}(g)$.

Step 2: Given a schedule profile, supplier $j$ allocate its endowment to manufacturers $x_{j}(t)=\left(x_{j}^{1}(t), x_{j}^{2}(t)\right)$. An allocation $x_{j}(t)$ is feasible if $\sum_{i} x_{j}^{i}(t) \leq \omega_{j}$, and $x_{j}^{i}(t) \equiv 0$ for all $i \notin L_{j}(g)$.

In Step 2, independently of the other supplier, given $g$ and $\left(t_{j}^{1}, t_{j}^{2}\right)$, each supplier $j$ maximizes his payoff function

$$
\Pi_{j}\left(x_{j}^{1}, x_{j}^{2}\right)=\sum_{i \in I} t_{j}^{i}\left(x_{j}^{i}(t)\right)
$$

Let $x_{j}^{*}(t)=\left(x_{j}^{1 *}(t), x_{j}^{2 *}(t)\right) \in \arg \max \Pi_{j}\left(x_{j}^{1}, x_{j}^{2}\right)$. Then, a payoff function for manufacturer $i$ is given by

$$
\Pi^{i}(t)=f^{i}\left(x_{1}^{i *}(t)+x_{2}^{i *}(t)\right)-\sum_{j \in J} t_{j}^{i}\left(x_{j}^{i *}(t)\right)
$$

Linear bidding and Equilibrium We focus on linear subgame perfect equilibria (linear SPEs), whereas the strategy space includes all non-linear bidding. A linear bidding strategy is defined as follows:

Definition 3 (Linear Bidding). A payment schedule from $i$ to $j, t_{j}^{i}\left(x_{j}^{i}\right)$, is linear if $t_{j}^{i}\left(x_{j}^{i}\right)$ satisfies for

\footnotetext{
${ }^{2}$ Let $-i$ and $-j$ be a manufacturer who is not $i$ and a supplier who is not $j$, respectively.
} 
price $p_{j}^{i}$

$$
t_{j}^{i}\left(x_{j}^{i}\right)=p_{j}^{i} x_{j}^{i}
$$

A strategy for manufacturer $i$ is linear bidding strategy if $t_{j}^{i}\left(x_{j}^{i}\right)$ is linear for all $j \in L^{i}(g)$, and a strategy profile is a linear SPE if the profile is a SPE in which all manufacturers adopt linear bidding strategies.

The linear SPEs are characterized as follows.

Proposition 2. For each $g \in G$, there exists a linear SPE with price vector $p^{*}$ such that for all $i$,

$$
p_{j}^{i *}(g)= \begin{cases}0 & \text { if } \eta_{j}(g)=0,1 \\ \tilde{p}(g) & \text { if } \eta_{j}(g)=2\end{cases}
$$

The corresponding linear SPE payoff vector $\Pi^{*}(g)$ is given by for all $i$,

$$
\Pi^{1 *}(g)=C S^{i}(g)+\sum_{j \in L^{i}(g), \eta_{j}(g)=1} P S_{j}(g)
$$

and for all $j$,

$$
\Pi_{j}^{*}(g)= \begin{cases}0 & \text { if } \eta_{j}=0,1 \\ P S_{j}(g) & \text { if } \eta_{j}=2 .\end{cases}
$$

Proof. When $\eta_{j}(g) \leq 1$ for all $j$ (i.e. $g \in\left\{g^{1}, g^{2}, g^{4}\right\}$ ), the bargaining game is the standard ultimatum bargaining. Thus, $p_{j}^{i *}(g)=0$. Each supplier $j$ allocates all endowments to the linked manufacturer, and each manufacturer obtains all profits in any linear SPE.

We then show that for any $g$ and any supplier $j$ with $\eta_{j}(g)=2, p_{j}^{1 *}(g)=p_{j}^{2 *}(g)$ in any linear SPE. Suppose $p_{j}^{1 *}(g)>p_{j}^{2 *}(g)$. Then, manufacturer 1 can deviates to decrease $p_{j}^{1}(g)=$ $p_{j}^{1 *}(g)-\epsilon$ for sufficiently small $\epsilon>0$.

When $g^{3}$ is given, there are two manufacturers and one supplier in the market. Consider a linear bidding strategy profile $t_{2}^{*}=\left(t_{2}^{1 *}, t_{2}^{2 *}\right)$ such that for any manufacturer $i=1,2$,

$$
p_{2}^{i *}=\tilde{p}\left(g^{3}\right)=\frac{d f^{i}}{d x^{i}}\left(\hat{x}^{i}\left(g^{3}\right)\right),
$$

and supplier 2 chooses $x_{2}^{*}=\left(\tilde{x}_{2}^{1}\left(g^{3}\right), \tilde{x}_{2}^{2}\left(g^{3}\right)\right)$. In this profile, $\left(\tilde{x}_{2}^{1}\left(g^{3}\right), \tilde{x}_{2}^{2}\left(g^{3}\right)\right),\left(\omega_{2}, 0\right)$, and $\left(0, \omega_{2}\right)$ are best replies for supplier 2 . If manufacturer 1 deviates to $p_{2}^{1 *}+\epsilon$ then supplier 2 chooses $\left(x_{2}^{1}, x_{2}^{2}\right)=\left(\omega_{2}, 0\right)$ and deviates to $p_{2}^{1 *}-\epsilon$ then supplier 2 chooses $\left(x_{2}^{1}, x_{2}^{2}\right)=\left(0, \omega_{2}\right)$ for any 
$\epsilon>0$. Both are not profitable since $\left(p_{2}^{1 *}, x_{2}^{1 *}\right)=\left(\tilde{p}_{2}^{1}\left(g^{3}\right), \tilde{x}_{2}^{1}\left(g^{3}\right)\right)$. Thus, this strategy profile constitutes a SPE, and the corresponding SPE payoff vector is $\Pi^{*}\left(g^{3}\right)$.

When $g^{5}$ is given, there are two manufacturers and two suppliers in the market. Consider an linear strategy profile $\left(\left(t_{1}^{1 *}, t_{2}^{1 *}\right), t_{2}^{2 *}\right)$ such that for manufacturer 1 ,

$$
\begin{aligned}
& p_{1}^{1 *}=0 \\
& p_{2}^{1 *}=\tilde{p}\left(g^{5}\right)=\frac{d f^{1}}{d x^{1}}\left(\tilde{x}^{1}\left(g^{5}\right)\right)
\end{aligned}
$$

and for manufacturer 2,

$$
p_{2}^{2 *}=\tilde{p}\left(g^{5}\right)=\frac{d f^{2}}{d x^{2}}\left(\tilde{x}^{2}\left(g^{5}\right)\right)
$$

Linear bidding $t_{1}^{1 *}\left(x_{1}^{1}\right)$ is optimal by $L_{1}\left(g^{5}\right)=\{1\}$, and $\left(t_{2}^{1 *}, t_{2}^{2 *}\right)$ constitutes a linear SPE since it is a linear SPE of $g^{3}$ given $x_{1}^{1}=\omega_{1}$. Hence, this profile constitutes a linear SPE and generates a payoff vector $\Pi^{*}\left(g^{5}\right)$.

When $g^{c}$ is given, consider a linear strategy profile $\left(\left(t_{1}^{1 *}, t_{2}^{1 *}\right),\left(t_{1}^{2 *}, t_{2}^{2 *}\right)\right)$ such that for manufacturer $i=1,2$ and supplier $j=1,2$,

$$
p_{j}^{i *}=\tilde{p}\left(g^{c}\right)=\frac{d f^{i}}{d x^{i}}\left(\tilde{x}^{i}\left(g^{c}\right)\right)
$$

and $\left(x_{j}^{1}\left(t^{*}\right), x_{j}^{2}\left(t^{*}\right)\right)=\left(\tilde{x}_{j}^{1}\left(g^{c}\right), \tilde{x}_{j}^{2}\left(g^{c}\right)\right)$ for supplier $j=1,2$. In this profile, $\left(\tilde{x}_{j}^{1}\left(g^{c}\right), \tilde{x}_{j}^{2}\left(g^{c}\right)\right)$ is a best reply for all $j$ and each supplier $j$ is indifferent to choose $\left(\tilde{x}_{j}^{1}\left(g^{c}\right), \tilde{x}_{j}^{2}\left(g^{c}\right)\right),\left(0, \omega_{j}\right)$, and $\left(\omega_{j}, 0\right)$. Thus, this strategy profile constitutes a linear SPE and generates a payoff vector $\Pi^{*}\left(g^{c}\right)$.

The above linear SPE is not a unique SPE, but the unique linear SPE.

Proposition 3. $\left(p^{*}, x^{*}\right)$ is the unique linear SPE.

Proof. Obviously, if $\eta_{j}(g)=1$, then $p_{j}^{i *}=0$ and $x_{j}^{i *}=\omega_{j}$ for all $i, j$ in any linear SPE. Thus, when $g^{1}, g^{2}$, or $g^{4}$ is given, there is a unique SPE.

Consider any $j$ with $\eta_{j}(g)=2$. Then, since $g \in\left\{g^{3}, g^{5}, g^{c}\right\}, p_{j}^{*}(g)=\tilde{p}(g)$. Suppose that there is a linear SPE $t=(p(g), x(g))$ with $p_{j}^{1}(g)=p_{j}^{2}(g)>\tilde{p}(g)$ for some $g$ and $j$. Consider a non-linear bidding $\bar{t}_{j}^{i}$ such that for some pair $\left(\bar{p}_{j}^{i}, \bar{y}_{j}^{i}\right)$,

$$
\bar{t}_{j}^{i}\left(x_{j}^{i}\right)= \begin{cases}\bar{p}_{j}^{i} x_{j}^{i} & \text { if } x_{j}^{i} \leq \bar{y}_{j}^{i} \\ \bar{p}_{j}^{i} \bar{y}_{j}^{i} & \text { if } x_{j}^{i}>\bar{y}_{j}^{i}\end{cases}
$$

By $\omega_{j}=\sum_{i} x_{j}^{i}(g)=\sum_{i} \tilde{x}_{j}^{i}(g)$, we obtain $x_{j}^{i *}(t) \geq \tilde{x}_{j}^{i}(g)$ for some $i$. Since $p_{j}^{i}(g)>\tilde{p}(g)$, it is profitable for such manufacturer $i$ to deviate $\bar{t}_{j}^{i}$ with $\left(\bar{p}_{j}^{i}, \bar{y}_{j}^{i}\right)=\left(p_{j}^{i}(g), x_{j}^{i}(g)-\epsilon\right)$ for some $\epsilon>0$. 
Next, suppose that there is a linear SPE $t=(p(g), x(g))$ with $p_{j}^{1}(g)=p_{j}^{2}(g)<\tilde{p}(g)$ for some $g$ and some $j$. Since $\tilde{p}(g)>p_{j}^{i}(g)$ for some $i$, it is profitable for such $i$ to deviate a non-linear schedule $\bar{t}_{j}^{i}$ with $\bar{p}_{j}^{i}=p_{j}^{i}+\epsilon$ and $\bar{y}_{j}^{i}$ such that $\frac{d f^{i}}{d x^{i}}\left(\bar{y}_{j}^{i}+x_{-j}^{i}(g)\right)=\bar{p}_{j}^{i}$. Therefore, $p_{j}^{i *}=\frac{d f^{i}}{d x^{i}}\left(\tilde{x}^{i}(g)\right)$ for all $j$ with $L_{j}(g)=\{1,2\}$ in any linear SPE.

Suppose that $\left(x_{j}^{1 *}(t), x_{j}^{2 *}(t)\right) \neq\left(\tilde{x}_{j}^{1}(g), \tilde{x}_{j}^{2}(g)\right)$. Then, there is a manufacturer with $x_{j}^{i *}(t)>$ $\tilde{x}_{j}^{i}(g)$. For such manufacturer $i$, it is a profitable to deviate $\bar{t}_{j}^{i}$ with $\bar{y}_{j}^{i}=\tilde{x}_{j}^{i}(g)$. Hence, $x_{j}^{*}(t)=$ $\left(\tilde{x}_{j}^{1}(g), \tilde{x}_{j}^{2}(g)\right)$ in any linear SPE.

By Proposition 2 and 3, we obtain the following corollaries, straightforwardly.

Corollary 1. The linear SPE allocation $x^{*}(g)$ is g-efficient for all $g \in G$.

Corollary 2. The linear SPE $\left(p^{*}(g), x^{*}(g)\right)$ is $g$-competitive if and only if $g=g^{c}$.

When $g^{c}$ is given, the set of feasible trading is the same as the set of feasible allocation in the nonnetworked market since all manufacturers can trade with all suppliers. In addition, manufacturers and suppliers are competitive since any manufacturer and any supplier have two links. Thus, the equilibrium allocation coincides with the Walrasian allocation. For any $g \in G \backslash\left\{g^{c}\right\}$, however, there always exists a supplier who has only one link. That supplier sells resources at price 0 in the linear SPE. Thus, the linear SPE is not $g$-competitive.

\section{Formation of Networked Market}

We have studied trading in the bargaining stage (the subgame after a network is formed). Next, we study a network formation game. We first introduce transaction cost to form links. Let $l^{i}(g)$ and $l_{j}(g)$ denote cost functions to form network $g$ for manufacturer $i$ and supplier $j$ respectively.

Assumption 2. Each link cost function $l^{i}$ and $l_{j}$ satisfy the following for all $i \in I$ and $j \in J$ :

$2.1 l^{i}(g)=\operatorname{l} \eta^{i}(g)$ and $l_{j}(g)=\operatorname{l} \eta_{j}(g)$, where $l>0$ is a constant.

$2.2 C S^{i}(g)-\operatorname{l\eta }(g)>0$ if $L^{i}(g) \neq \varnothing$ and $P S_{j}(g)-\ln (g)>0$ if $L_{j}(g) \neq \varnothing$ for all $g$.

$2.3 S W\left(g^{5}\right)>\max \left\{S W\left(g^{2}\right), S W\left(g^{3}\right)\right\}$.

Assumption 2.1 is the standard linearity assumption of link cost function. Each link $i j$ is formed at strictly positive cost $l$ for both manufacturer $i$ and supplier $j$. By Assumption 2.2, the $g$-competitive allocation is more profitable than no trading for any firms and any network. This assumption implies that $S W\left(g^{e}\right)<S W\left(g^{1}\right)<S W\left(g^{4}\right)$. Assumption 2.3 implies that $g$ is inefficient when there is an agent who does not participate the networked market.

Then, we define a social welfare function as

$$
S W(g)=\sum_{i}\left[\Pi^{i *}(g)-\ln \eta^{i}(g)\right]+\sum_{j}\left[\Pi_{j}^{*}(g)-\ln \eta_{j}(g)\right] .
$$


The efficient network is $\hat{g} \in \arg \max _{g} S W(g)$, and the efficient allocation of resources is $\hat{x}(\hat{g})$.

If $g=g^{c}$, then there is an efficient allocation $\hat{x}\left(g^{c}\right)$ such that there is at least one link ij with $\hat{x}_{j}^{i}\left(g^{c}\right)=0$. Thus, at least one type of $g^{5}$ satisfies $\hat{x}_{j}^{i}\left(g^{c}\right)=\hat{x}_{j}^{i}\left(g^{5}\right)$ for any link $i j \in g^{c}$. For such $g^{5}$, $S W\left(g^{c}\right)<S W\left(g^{5}\right)$. Therefore, for any economy $\left(f^{1}, f^{2}, \omega_{1}, \omega_{2}\right)$,

$$
\hat{g} \in\left\{g^{4}, g^{5}\right\}
$$

In particular, if all manufacturers and suppliers are symmetric $\left(f^{1}=f^{2}\right.$ and $\left.\omega_{1}=\omega_{2}\right)$, then $g^{4}=\hat{g}$.

\subsection{Link-Announcement Game}

We adopt the link-announcement game (Jackson [8]) as a network formation game. All manufacturers and suppliers announce a set of agents with whom they want to form links simultaneously. We denote a (pure) strategy of manufacturer $i$ by $s^{i}=\left(s^{i j}\right)_{j \in S}$, and a strategy of supplier $j$ by $s_{j}=\left(s_{i j}\right)_{i \in B}$, where $s^{i j}, s_{i j} \in\{+,-\}$. If $s^{i j}=+$ then manufacturer $i$ wants to link with supplier $j$ and if $s_{i j}=+$ then supplier $j$ wants to link with manufacturer $i$. A link $i j$ is formed if and only if both manufacturer $i$ and supplier $j$ agree with forming link $i j$, i.e. $i j \in g(s)$ if and only if $s^{i j}=s_{i j}=+$.

Thus, payoffs of manufacturer $i$ and supplier $j$ are given by

$$
\begin{aligned}
& \phi^{i}(g(s))=\Pi^{i}(t(g(s)), x(g(s)))-\operatorname{l\eta }^{i}(g(s)) \\
& \phi_{j}(g(s))=\Pi_{j}(t(g(s)), x(g(s)))-\operatorname{l} \eta_{j}(g(s)) .
\end{aligned}
$$

We assume that all agents play a linear SPE after any network is formed. A profile $(s, t(g), x(g))$ is a Nash equilibrium if $(t(g), x(g))$ is a linear SPE in the bargaining game for all $g$, and for all $i$ and $j$,

$$
\begin{aligned}
& \phi^{i}(g(s)) \geq \phi^{i}\left(g\left(s^{\prime i}, s_{-i}\right)\right), \\
& \phi_{j}(g(s)) \geq \phi_{j}\left(g\left(s_{j}^{\prime}, s_{-j}\right)\right) .
\end{aligned}
$$

A network $g$ is Nash stable (NS) if there exists a Nash equilibrium with $g(s)=g$.

It is well-known that the Nash stability is a weak concept for bilateral network formation games. There are many NS networks (e.g. the empty network is always Nash stable for all network formation games). A standard refinement of Nash stability proposed by Bloch and Jackson [1] is the pairwise Nash stability (PNS). In addition to the Nash stability, we demands that there is no profitable pairwise deviation.

Definition 4. A network $g$ is pairwise Nash stable if it is Nash stable and there does not exist a link 
ij $\notin g(s)$ such that

$$
\begin{aligned}
& \phi^{i}(g(s) \cup\{i j\}) \geq \phi^{i}(g(s)) \\
& \phi_{j}(g(s) \cup\{i j\}) \geq \phi_{j}(g(s))
\end{aligned}
$$

with strict inequality for one of two agents.

\subsection{Stable Market Structure}

First, suppose that $l=0$ instead of Assumption 2.1. Then, Proposition 2 implies that the complete network $g^{c}$ is the unique PNS network since forming any link $i j$ is profitable for $i$ and $j$, i.e. $\Pi^{i *}(g \cup\{i j\}) \geq \Pi^{i *}(g)$ and $\Pi_{j}^{*}(g \cup\{i j\}) \geq \Pi_{j}^{*}(g)$ with strict inequality one of two agents for all $g$ and $i j \notin g$. Therefore, if there is no transaction cost, the unique stable market structure is the complete networked market resulting in the Walrasian outcome.

Hereafter, we assume that $l>0$. The next proposition shows that any efficient network and the complete network are not Nash stable.

Proposition 4. In the link-announcement game,

(i) The network $g^{e}$ and $g^{3}$ are PNS.

(ii) The other networks $g^{1}, g^{2}, g^{4}, g^{5}$, and $g^{c}$ are not NS.

Proof. Part i: By Proposition 2, $\Pi_{j}^{*}\left(g^{e} \cup\{i j\}\right)=0$ for any $i j$ and $j$. Then, there is no link $i j$ satisfying $\phi_{j}\left(g^{e} \cup\{i j\}\right)-\phi_{j}\left(g^{e}\right)=-l \geq 0$. Hence, $g^{e}$ is PNS.

For $g^{3}$, since all manufacturers obtain non-negative payoffs and have only one link, there is no incentive to cut a link for manufacturers. If supplier 2 who obtain non-negative payoff cut any one of links then 2's payoff is $-l$ and if supplier 2 cut both then 2's payoff is 0 . Hence, supplier 2 also has no incentive to cut links. By Proposition $2, \Pi_{1}^{*}\left(g^{3} \cup\{11\}\right)=\Pi_{1}^{*}\left(g^{5}\right)=\Pi_{1}^{*}\left(g^{3}\right)=0$. Then, supplier 1 has no incentive to form link 11 since $\phi_{1}\left(g^{3} \cup\{11\}\right)-\phi_{1}\left(g^{3}\right)=-l<0$.

Part ii: If $C S^{i}\left(g^{c}\right)-2 l \geq C S^{i}\left(g^{c} \backslash\{i j\}\right)-l \geq 0$ holds for all $i, j$, there is no incentive for manufacturer $i$ to cut any links in $L^{i}\left(g^{c}\right)$. However, there is an efficient allocation with $\hat{x}_{j}^{i}\left(g^{c}\right)=0$ for some $i j$. Let $\hat{x}_{1}^{2}\left(g^{c}\right)=0$. This implies that $\Pi^{2 *}\left(g^{c}\right)=\Pi^{2 *}\left(g^{5}\right)$. Hence, cutting the link 21 is profitable for the manufacturer 2 .

Similarly, for other networks $g^{1}, g^{2}, g^{4}$, or $g^{5}$, supplier 1 obtains no profit. Thus, supplier 1 deviates by cutting a link.

An under-investment problem Networks $g^{e}, g^{3}$ never become efficient network by (3). This inefficiency is caused by a hold-up problem (Hart and Moore [7]). When a pair of a manufacturer and a supplier jointly invest in a link (a relation-specific asset), cost of forming link is not contractible 
and sunk in the bargaining stage. Thus, under-investment arises for suppliers since they have less bargaining power.

\section{Side-payment Contracts}

In the previous section, we assume that no side-payment contract contingent on links is available for all firms. They sometimes, however, can write some simple contingent contract with sidepayments. This section studies a network formation game with direct side-payments.

Network Formation Game with Direct Side-Payments Bloch and Jackson [2] propose a network formation game which allows direct side-payments when agents form a network. In their network formation game with direct side-payments, every manufacturer $i$ and supplier $j$ simultaneously announce a vector of transfers $\tau^{i} \in \mathbb{R}^{s}, \tau_{j} \in \mathbb{R}^{b}$. All manufacturers and suppliers can only transfer to form links with which they involved. We denote the element of $\tau^{i}$ by $\tau^{i j} \in \mathbb{R}$, which represents transfer that manufacturer $i$ proposes to form link $i j$ and $\tau_{j}$ by $\tau_{i j} \in \mathbb{R}$, which represents transfer that supplier $j$ proposes to form link $i j$. Link $i j$ is formed if and only if $\tau^{i j}+\tau_{i j} \geq 0$. A network $g(\tau)=\left\{i j \mid \tau^{i j}+\tau_{i j} \geq 0\right\}$, where $\tau=\left(\tau^{1}, \tau^{2}, \tau_{1}, \tau_{2}\right)$ is a profile of announced vectors of transfers.

The payoffs for manufacturer $i$ and supplier $j$ are defined by

$$
\begin{aligned}
& \Phi^{i}(g(\tau))=\phi_{i}(g(\tau))-\sum_{i m \in g(\tau)} \tau^{i m} \\
& \Phi_{j}(g(\tau))=\phi_{j}(g(\tau))-\sum_{j k \in g(\tau)} \tau_{j k} .
\end{aligned}
$$

Given $\tau$, let $\tau_{-i j}$ indicate the profile of transfers found simply by deleting $\tau^{i j}$ and $\tau_{i j}$. The stability concepts in the network formation game with direct transfer are defined in the same manner.

Definition 5. A strategy profile $(\tau, t(g), x(g))$ is a Nash equilibrium if $(t(g), x(g))$ is a linear SPE in the bargaining game for all $g$, and for all $i$ and $j$,

$$
\begin{aligned}
& \Phi^{i}(g(\tau)) \geq \Phi^{i}\left(g\left(\tau^{\prime i}, \tau^{-i}\right)\right) \\
& \Phi_{j}(g(\tau)) \geq \Phi_{j}\left(g\left(\tau_{j}^{\prime}, \tau_{-j}\right)\right) .
\end{aligned}
$$

A network $g$ is Nash stable with direct transfer (NSd) if there is a Nash equilibrium with $g(\tau)=g$. A network $g$ is pairwise Nash stable with direct transfer (PNSd) if it is NSd and there exists no ij $\notin g(\tau)$ such that for all $\tau^{\prime i j}, \tau_{i j}^{\prime}$

$$
\Phi^{i}\left(g\left(\tau_{-i j}, \tau^{\prime i j}, \tau_{i j}^{\prime}\right)\right)+\Phi_{j}\left(g\left(\tau_{-i j}, \tau^{\prime i j}, \tau_{i j}^{\prime}\right)\right)>\Phi^{i}(g(\tau))+\Phi_{j}(g(\tau))
$$

Lemma 1. In the network formation game with direct transfer, any efficient network $\hat{g}$ is NSd but not PNSd. 
Furthermore, the set of PNSd networks is empty or $\left\{g^{c}\right\}$.

Proof. First note that $\hat{g} \in\left\{g^{4}, g^{5}\right\}$. Suppose $\hat{g}=g^{4}$. Since $C S^{i}(\{i j\})+P S_{j}(\{i j\}) \geq 2 l$ for all $i, j$, there is a profile $\tau$ such that no deviation to cut any link is profitable. Hence, there is a Nash equilibrium $\tau$ such that $g(\tau)=g^{4}$. Thus, $g^{4}$ is NSd when $\hat{g}=g^{4}$.

Next, suppose $\hat{g}=g^{5}$. Then, $C S^{i}\left(g^{5}\right)=C S^{i}\left(g^{c}\right)$ and $P S_{j}\left(g^{5}\right)=P S_{j}\left(g^{c}\right)$ since $\hat{x}\left(g^{5}\right)=\hat{x}\left(g^{c}\right)$ for all $i$ and $j$. Consider any profile $\tau$ satisfying $\tau^{11}=-\tau_{11}=l, \tau^{12}=-\tau_{12}=-l, \tau^{21}+\tau_{21}<0$, and $\tau^{22}=-\tau_{22}=C S^{2}\left(g^{5}\right)-l$. Then, $g(\tau)=g^{5}$. Since $\Phi^{2}(g(\tau))=\Phi_{1}(g(\tau))=0$, there is no profitable deviation for manufacturer 2 and supplier 1 . Furthermore, manufacturer 1 and supplier 2 have no profitable deviation since

$$
\begin{aligned}
& C S^{1}\left(g^{c}\right)+P S_{1}\left(g^{c}\right)-2 l \geq \max \left[C S^{1}\left(g^{3}\right), C S^{1}\left(g^{4}\right)+P S_{1}\left(g^{4}\right)-2 l\right] \\
& C S^{2}\left(g^{c}\right)+P S_{2}\left(g^{c}\right)-4 l \geq C S^{2}\left(g^{c}\right)-2 l
\end{aligned}
$$

Hence, $g^{5}$ is NSd.

However, since $\Pi^{1 *}\left(g^{5}\right)+\Pi_{2}^{*}\left(g^{5}\right)-\Pi^{1 *}\left(g^{4}\right)-\Pi_{2}^{*}\left(g^{4}\right)=C S^{1}\left(g^{5}\right)+\sum_{j} P S_{j}\left(g^{5}\right)-C S^{1}\left(g^{4}\right)-$ $P S_{1}\left(g^{4}\right)>2 l$ and $\Pi^{2 *}\left(g^{c}\right)+\Pi_{1}^{*}\left(g^{c}\right)-\Pi^{2 *}\left(g^{5}\right)-\Pi_{1}^{*}\left(g^{5}\right)=C S^{2}\left(g^{c}\right)+P S_{1}\left(g^{c}\right)-C S^{2}\left(g^{5}\right)>$ $2 l$ by Assumption 2.2, $g^{4}$ and $g^{5}$ are not pairwise Nash stable. Hence, any efficient network is not pairwise Nash stable. By the same procedure, we can show that $g^{e}, g^{1}, g^{2}$, and $g^{3}$ are not pairwise Nash stable.

Next, we show that the competitive outcome in the complete networked market $g^{c}$ (the Walrasian outcome) is the unique pairwise Nash stable outcome when the link cost $l$ is small.

Proposition 5. Suppose that $P S_{j}\left(g^{c}\right) \geq 4 l$ for all $j$. In the network formation game with direct side-payments, the complete network $g^{c}$ is the unique PNSd network.

Proof. First, we construct $\tau$ with $g(\tau)=g^{c}$ such that there is no incentive to cut any link. Consider a profile $\tau$ such that $\tau^{i j}=-\tau_{i j}=-l$ for all $i j$. Then the manufacturer $i$ has no incentive to cut any link since $C S^{i}\left(g^{c}\right) \geq C S^{i}\left(g^{c} \backslash\{i j\}\right)$ for all $i j$ and $i^{\prime}$ s transfer from $j,-\tau^{i j}$, is equal to cost of maintaining link. Also, the supplier $j$ has no incentive to cut any link since $\Pi_{j}^{*}\left(g^{c}\right)-2 l-\sum \tau_{i j}=$ $P S_{j}\left(g^{c}\right)-4 l \geq 0$. Hence, $g^{c}$ is a PNSd network. By Lemma 1 , it is the unique PNSd network.

In constrast, when $l$ is high, there is no pairwise Nash equilibrium with direct side-payments for some economy $\left(f^{1}, f^{2}, \omega_{1}, \omega_{2}\right)$. There is a supplier who deviates to cut a link in the complete network. We consider a symmetric economy $\left(f^{1}, f^{2}, \omega_{1}, \omega_{2}\right)$ where $f^{1}=f^{2}$ and $\omega_{1}=\omega_{2}$.

Proposition 6. Suppose that an economy is symmetric and PS $\left(g^{c}\right)<4 l$. Then, $g^{c}$ is not NSd, and there is no PNSd network.

Proof. When all agents are symmetric, $C S^{i}\left(g^{c}\right)=C S^{i}\left(g^{5}\right)$ for any $i$ and any type of $g^{5}$. Thus, in $g^{c}$, there is no incentive for any manufacturer $i$ to cut link $i j$ if and only if $\tau^{i j} \leq-l$ for all $j$. However, 
since $P S\left(g^{c}\right)<4 l$, such transfer $\left(\tau_{1 j}, \tau_{2 j}\right)$ is not profitable for any supplier $j$. Hence, each supplier has an incentive to cut a link, and $g^{c}$ is not NSd. Since any other network is not pairwise Nash stable by Lemma 1, there is no PNSd network.

An over-investment problem A new hold-up problem arises in the network formation with direct side-payments. Suppose that link cost is low. By Lemma 1, the complete networked market is uniquely pairwise Nash stable. Each suppliers $j$ has an incentive to form two links $1 j$ and $2 j$ by transferring link cost $l$ to both manufacturers for $j$ 's surplus $P S_{j}$ when $4 l<P S_{j}$. Since the Walrasian outcome is an equilibrium outcome in the bargaining subgame when the market is completely networked, it is supported by the network formation game with direct side-payments. However, it is not efficient by (3). There is a link $i j$ in which there is no trading in the complete networked market $\left(\hat{x}_{j}^{i}\left(g^{c}\right)=0\right)$. Therefore, there is an over-investment problem due to excess link formation.

\section{Large Economy}

We have shown that the stable allocation is inefficient in the small networked market consisting of two manufacturers and two suppliers. This section studies a large symmetric networked market. Suppose that an economy consists of $n$ symmetric manufacturers and $n$ symmetric suppliers $(n \geq 3)$. All manufacturers have the same profit function $f$ and all suppliers have the same endowment $\omega$. Then, any efficient network has the same architecture as $\hat{g}=\{11,22, \ldots, n n\}$. We first characterize linear SPEs in Stage 2.

Lemma 2. Fix $g$. In any linear SPE $\left(p^{*}(g), x^{*}(g)\right)$, price vector $p^{*}(g)$ is given by for any $j$,

$$
\max _{i \in L_{j}(g)} p_{j}^{i *}(g)= \begin{cases}0 & \text { if } \eta_{j}(g)=0,1 \\ \tilde{p}_{j}(g) & \text { if } \eta_{j}(g) \geq 2\end{cases}
$$

Proof. It is obvious that $p_{j}^{i *}(g)=0$ for all $j$ with $\eta_{j}(g) \leq 1$.

Suppose that $\max _{i \in L_{j}(g)} p_{j}^{i *}(g)>\tilde{p}_{j}(g)$ for some $j$. Then, there is a manufacturer $i$ with $x_{j}^{i *}(g) \geq \tilde{x}_{j}^{i}(g)>0$ since $\sum_{i} x_{j}^{i *}(g)=\sum_{i} \tilde{x}_{j}^{i}(g)=\omega_{j}$. By condition (i) in Definition 1, it is profitable for such $i$ to bid a non-linear schedule $\bar{t}$ such that for $\epsilon>0$,

$$
\bar{t}_{j}^{i}\left(x_{j}^{i}\right)= \begin{cases}p_{j}^{i *}(g) x_{j}^{i} & \text { if } x_{j}^{i} \leq x_{j}^{i *}(g)-\epsilon \\ p_{j}^{i *}(g)\left(x_{j}^{i *}(g)-\epsilon\right) & \text { if } x_{j}^{i}>x_{j}^{i *}(g)-\epsilon .\end{cases}
$$

Suppose that $\max _{i \in L_{j}(g)} p_{j}^{i *}(g)<\tilde{p}_{j}(g)$ for some $j$. Then, there is a manufacturer $i$ who deviate 
to non-linear schedule such that for $\epsilon>0$ and $\bar{y}_{j}^{i}$ such that $\frac{d f^{i}}{d x^{i}}\left(\bar{y}_{j}^{i}+x_{-j}^{i}(g)\right)=p_{j}^{i *}+\epsilon$,

$$
\bar{t}_{j}^{i}\left(x_{j}^{i}\right)= \begin{cases}\left(p_{j}^{i *}(g)+\epsilon\right) x_{j}^{i} & \text { if } x_{j}^{i} \leq \bar{y}_{j}^{i} \\ \left(p_{j}^{i *}(g)+\epsilon\right)\left(x_{j}^{i *}(g)-\epsilon\right) & \text { if } x_{j}^{i}>\bar{y}_{j}^{i}\end{cases}
$$

Obviously, if no side-payment is available, then the efficient network $\hat{g}$ is not pairwise Nash stable. Since any supplier $j$ has only one link, it is profitable for $j$ to cut his link. Even if simple contingent contracts are available, the efficient network $\hat{g}$ is not pairwise Nash stable.

Proposition 7. Any efficient network $\hat{g}$ is not PNSd in either the link announcement game and the network formation game with direct side-payments.

Proof. First, consider the link announcement game. Then, $p_{j}^{i *}(\hat{g})=0$ for all $j$. Thus, it is profitable to cut $j$ 's link for any $j$.

Second, consider the network formation game with the direct side-payments. When $g(\tau)=\hat{g}$, $p_{j}^{i *}(g(\tau))=0$. Since $P S_{j}(g) \geq 2 l$ for all $j$, it is profitable to deviates to form a new link $i j \notin \hat{g}$ by transferring $l$ to manufacturer $i$ for any supplier $j$.

Therefore, efficient trading is not pairwise Nash stable in the large market. The origins are the same as $n=2$ : When no side-payments are available, the under-investment for suppliers arises. When side-payments are available, the inefficiency is caused by the over-investment problem.

When $n=2$ and link cost $l$ is low, the complete network is PNSd. However, the complete network is not PNSd whenever $l>0$ in the large market with $n \geq 3$. For any $j$ and any $g$ with $\eta_{j}(g) \geq 2, \max _{i} p_{j}^{i *}(g)=\tilde{p}_{j}(g)$. By symmetry, $\tilde{p}_{j}\left(g^{c}\right)=\tilde{p}_{j}\left(g^{c} \backslash\{i j\}\right)$ for any $i j \in g^{c}$. Thus, any $j$ has an incentive to cut at least one link in $g^{c}$ when $n \geq 3$.

\section{Concluding Remarks}

We have studied decentralized trading in the strategically formed networked market between manufacturers and suppliers. In contrast to the centralized trading in a buyer-seller networked market, we show that any efficient network is not pairwise Nash stable, regardless of the presence of the side-payment contracts. When no side-payment contract is available, suppliers under-invest in links. When side-payments are available, the complete network resulting in the Walrasian equilibrium outcome is the unique pairwise Nash stable network if link cost is low. However, it is not efficient since the firms over-invest in links. Therefore, the efficient trading does not occur when firms strategically form a trading network.

The study has two limitations. First, it is not known whether the same results holds in a large market with heterogeneous firms. Shirata [12] further studies an allocation problem in a two-sided 
networked market with asymmetric multi firms given a network. Second, we only consider a noside-payment contract and a simple side-payment contract. Some complex or complete contracts may solve the inefficiency in the networked market.

\section{References}

[1] F. Bloch and M. O. Jackson. Definitions of equilibrium in network formation games. International Journal of Game Theory, 34(3):305-318, 2006. 10

[2] F. Bloch and M. O. Jackson. The formation of networks with transfers among players. Journal of Economic Theory, 133(1):83-110, Mar. 2007. 12

[3] R. H. Coase. The Firm, the Market, and the Law. Univ of Chicago Pr (Tx), reprint edition, Feb. 1990. ISBN 0226111016. 2

[4] M. Corominas-Bosch. Bargaining in a network of buyers and sellers. Journal of Economic Theory, 115(1):35-77, 2004. 1

[5] D. Gale. Strategic Foundations of General Equilibrium: Dynamic Matching and Bargaining Games. Cambridge University Press, 1 edition, Aug. 2000. ISBN 0521644100. 2

[6] D. Gale and H. Sabourian. Complexity and competition. Econometrica, 73(3):739-769, 2005. 2

[7] O. Hart and J. Moore. Incomplete contracts and renegotiation. Econometrica, 56(4):755-785, July 1988. 11

[8] M. O. Jackson. Social and Economic Networks. Princeton Univ Pr, Sept. 2008. ISBN 0691134405. 10

[9] R. E. Kranton and D. F. Minehart. Competition for goods in buyer-seller networks. Review of Economic Design, 5(3):301-331, 2000. 1

[10] R. E. Kranton and D. F. Minehart. A theory of Buyer-Seller networks. The American Economic Review, 91(3):485-508, June 2001. 1

[11] A. Rubinstein and A. Wolinsky. Decentralized trading, strategic behaviour and the walrasian outcome. The Review of Economic Studies, 57(1):63-78, 1990. 2

[12] Y. Shirata. First price package auction with many traders. mimeo, 2010. 15

[13] O. E. Williamson. The Economic Intstitutions of Capitalism. Free Press, Oct. 1998. ISBN 068486374X. 2 